\title{
Tunneling effect of the spin-2 Bose condensate driven by external magnetic fields
}

\author{
Zhao-xian $\mathrm{Yu}^{1}$, Zhi-yong Jiao ${ }^{1,2}$ \\ 1. Department of Applied Physics, University of Petroleum (East China), \\ Dongying 257061, Shandong Province, P.R.China \\ 2. Photonics Center, College of Physics, Nankai University, Tianjin 300071, P.R.China
}

\begin{abstract}
In this paper, we have studied tunneling effect of the spin-2 Bose condensate driven by external magnetic field. We find that the population transfers among spin- 0 and spin- \pm 1 , spin- 0 and spin- \pm 2 exhibit the step structure under the external cosinusoidal magnetic field respectively, but there do not exist step structure among spin- \pm 1 and spin- \pm 2 . The tunneling current among spin- \pm 1 and spin- \pm 2 may exhibit periodically oscillation behavior, but among spin-0 and spin- \pm 1 , spin- 0 and spin- \pm 2 , the tunneling currents exhibit irregular oscillation behavior.
\end{abstract} PACS numbers: 75.45.+j, 03.75.Fi, 05.30.Jp

Key words: Tunneling, population transfer, spinor Bose condensate.

\section{Introduction}

Recent advance of experimental techniques on Bose-Einstein condensate (BEC) prompts us to closely and seriously look into theoretical possibilities which were mere imagination for theoreticians in this field. This is particularly true for spinor BEC where all hyperfine states of an atom Bose-condensed simultaneously, keeping these "spin" states degenerate and active. Recently, Barrett et al [1] have succeeded in 
cooling $R b^{87}$ with the hyperfine state $F=1$ by all optical methods without resorting to a usual magnetic trap in which the internal degrees of freedom is frozen. Since the spin interaction of the $R b^{87}$ atomic system is ferromagnetic, based on the refined calculation of the atomic interaction parameters by Klausen et al [2], we now obtain concrete examples of the three-component spinor BEC $\left(F=1, m_{F}=1,0,-1\right)$ for both antiferromagnetic $\left(\mathrm{Na}^{23}\right)[3]$ and ferromagnetic interaction cases. In the present spinor $\mathrm{BEC}$ the degenerate internal degrees of freedom play an essential role to determine the fundamental physical properties. There is a rich variety of topological defect structures, which are already predicted in the earlier studies $[4,5]$ on the spinor BEC. Law et al [6] constructed an excellent algebraic representation of the $F=1$ BEC Hamiltonian to study the exact many-body states, and found that spin-exchange interactions cause a set of collective dynamic behavior of BEC. Since the spinor BEC appears feasible by using the $F=2$ multiplet of bosons, it is necessary to investigate the groundstate structure and magnetic response of $F=2$ spinor BEC. Recently, Ciobanu et al [7] generalized the approach for the $F=1$ spinor BEC to study the ground state structure of the $F=2$ spinor BEC. They found that there are three possible phases in zero magnetic field, which are characterized by a pair of parameters describing 
the ferromagnetic order and the formation of singlet pairs. From current estimates of scattering lengths, they also found that the spinor BEC's of $R b^{87}$ and $N a^{23}$ have a polar ground state, whereas those of $R b^{85}$ and $R b^{83}$ are cyclic and ferromagnetic, respectively. Koashi et al [8] studied the exact eigenspectra and eigenstates of $F=2$ spinor BEC. They found that, compared to $F=1$ spinor BEC, the $F=2$ spinor BEC exhibits an even richer magnetic response due to quantum correlations among three bosons. Recently, Zhang et al [9] studied dynamic response of the $F=2$ spinor BEC under the influence of external magnetic fields, they found that when the frequency and the reduced amplitude of the longitudinal magnetic field are related in a specific manner, the population of the initial spin-0 state will be dynamically localized during time evolution. In this paper, we shall investigate the tunneling effect of the spin-2 Bose condensate driven by external magnetic fields.

\section{$2 \quad$ Model}

We consider the $F=2$ spinor BEC subject to a spatial weak uniform magnetic fields which consist of longitudinal and transverse components. Without loss of generality, the transverse direction of the field is chosen to be along the $x$ axis, i.e., $\hat{B}(t)=$ 
$B_{l}(t) \hat{z}+B_{x} \hat{x}$. In such a case, the second-quantized Hamiltonian of the system is [9]

$$
\begin{gathered}
H=H_{0}+H_{B}, \\
H_{0}=\frac{c_{1}}{2} \hat{F} \cdot \hat{F}+\frac{2 c_{2}}{5} \hat{S}_{+} \hat{S}_{-}, \\
H_{B}=-\mu_{B} g_{f} B_{l}(t)\left(\hat{a}_{2}^{\dagger} \hat{a}_{2}+\hat{a}_{1}^{\dagger} \hat{a}_{1}-\hat{a}_{-1}^{\dagger} \hat{a}_{-1}-\hat{a}_{-2}^{\dagger} \hat{a}_{-2}\right) \\
-\mu_{B} g_{f} B_{x}\left(\hat{a}_{2}^{\dagger} \hat{a}_{1}+\sqrt{\frac{3}{2}} \hat{a}_{1}^{\dagger} \hat{a}_{0}+\sqrt{\frac{3}{2}} \hat{a}_{0}^{\dagger} \hat{a}_{-1}+\hat{a}_{-1}^{\dagger} \hat{a}_{-2}+H . c .\right),
\end{gathered}
$$

here, the $5 \times 5$ spin matrices $\hat{F}_{i}=\hat{a}_{\alpha}^{\dagger}\left(F_{i}\right)_{\alpha \beta} \hat{a}_{\beta}(i=x, y, z), \hat{S}_{+}=\hat{S}_{-}^{\dagger}=\left(\hat{a}_{0}^{\dagger}\right)^{2} / 2-\hat{a}_{1}^{\dagger} \hat{a}_{-1}^{\dagger}+$ $\hat{a}_{2}^{\dagger} \hat{a}_{-2}^{\dagger}$, and $c_{i}=\left(\overline{c_{i}} \int d \vec{r}|\phi|^{4}\right) \cdot \overline{c_{0}}, \overline{c_{1}}$, and $\overline{c_{2}}$ are related to scattering lengths $a_{0}, a_{2}$, and $a_{4}$ of the two colliding bosons.

Similar to Ciobanu et al [7], mean-field approximation is used such that the field operators $\hat{a}_{\alpha}$ are replaced by $c$ numbers $a_{\alpha}=\sqrt{P_{\alpha}} e^{i \theta_{\alpha}}$, where $P_{\alpha}=N_{\alpha} / N$ is the population in spin $\alpha$, and $\theta_{\alpha}$ the phase of wave function $a_{\alpha}$. Furthermore, since this paper deals with the quantum coherent behavior of the system under the influence of the external magnetic fields, we assume that the initial spin state of the BEC is the eigenstate in the absence of external fields, then contribution from Hamiltonian (2) is a constant energy shift and can be neglected in the study of the dynamics. The semiclassical equations of motion in Heisenberg representation can be derived from the 
Hamiltonian $H_{B}$ (here, the state vector $a=\left(a_{2}, \ldots, a_{-2}\right)^{T}$ is introduced)

$$
i \dot{a}=H_{e f f}(t) a
$$

where

$$
H_{e f f}(t)=-b_{l}(t) F_{z}-b_{x} F_{x}
$$

Here $b_{l}(t)=\mu_{B} g_{f} B_{l}(t)$ and $b_{x}=\mu_{B} g_{f} B_{x}$. In this paper, we consider the case that the system begins with unperturbed spin-0 state $a(0)=(0,0,1,0,0)^{T}$. If considering the case that the transverse magnetic field $b_{x}$ is weak, one can get the time evolution of the population of spin- $\alpha$ state as $[9]$

$$
P_{\alpha}(t)=\left|a_{\alpha}(t)\right|^{2}=\left|\sum_{\beta=-2}^{2} d_{\beta \alpha}^{2}(\pi / 2) d_{\beta 0}^{2}(\pi / 2) \exp \left(-i \alpha \int_{0}^{t} b_{l}(\tau) d \tau\right) e^{i \beta \lambda}\right|^{2}
$$

where

$$
\begin{gathered}
\lambda=\sqrt{\left[\int_{0}^{t} b_{x} \cos \left[\int_{0}^{\tau} b_{l}(\tau) d \tau\right] d \tau\right]^{2}+\left[\int_{0}^{t} b_{x} \sin \left[\int_{0}^{\tau} b_{l}(\tau) d \tau\right] d \tau\right]^{2}}, \\
d_{\beta \alpha}^{2}(\theta)=<2 \beta\left|e^{-i \theta F_{y}}\right| 2 \alpha>(\beta, \alpha=2, \ldots,-2) .
\end{gathered}
$$

From figures 1-3, we find that the population transfers among spin- 0 and spin- \pm 1 , spin- 0 and spin- \pm 2 exhibit the step structure under the external cosinusoidal magnetic field $b_{l}(t)=b \cos (\omega t)$ respectively, but there do not exist step structure among spin- \pm 1 and spin- \pm 2 . 


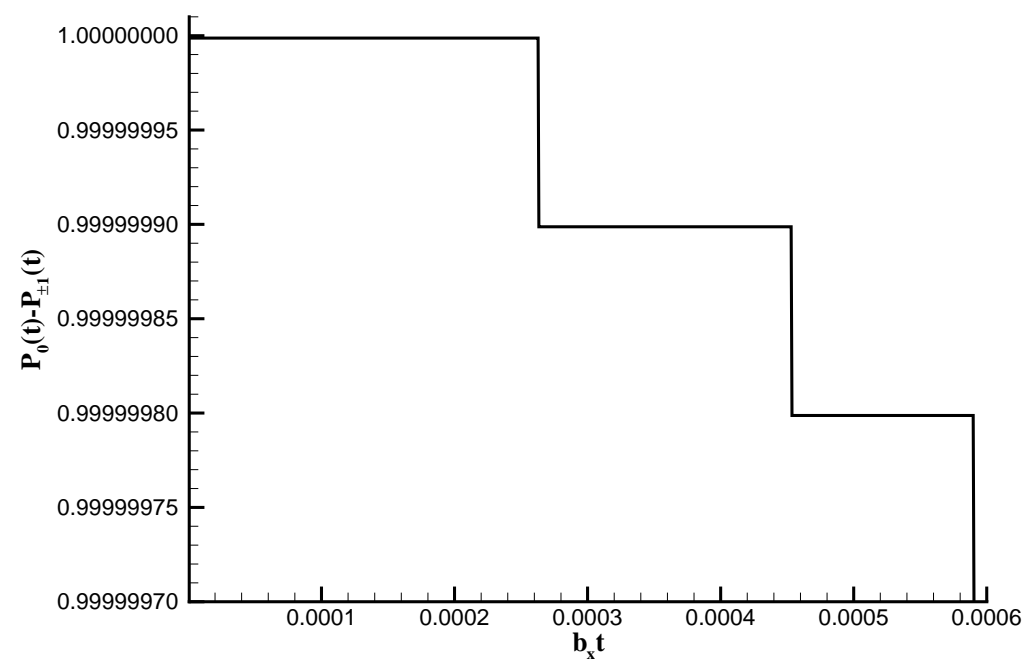

Figure 1: Time evolution of population transfer $P_{0}(t)-P_{ \pm 1}(t)$ with an external cosinusoidal magnetic field, $b_{x} / \omega=0.4, b / \omega=0.8$.

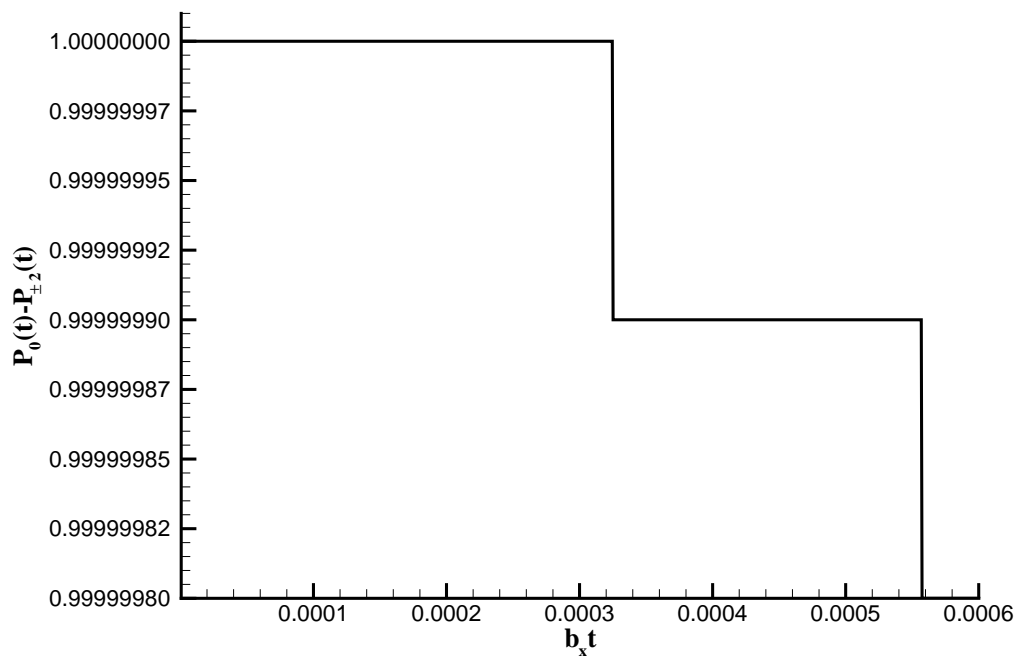

Figure 2: Time evolution of population transfer $P_{0}(t)-P_{ \pm 2}(t)$ with an external cosinusoidal magnetic field, $b_{x} / \omega=0.4, b / \omega=0.8$. 


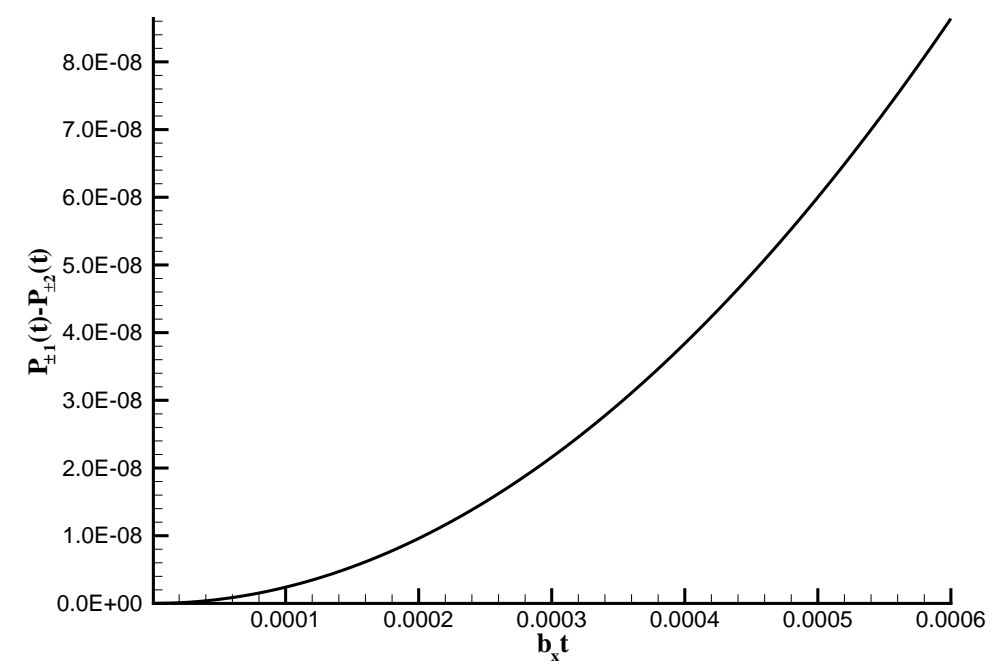

Figure 3: Time evolution of population transfer $P_{ \pm 1}(t)-P_{ \pm 2}(t)$ with an external cosinusoidal magnetic field, $b_{x} / \omega=0.4, b / \omega=0.8$.

\section{Tunneling effect of the spin-2 Bose condensate driven by external magnetic fields}

In this section, we shall study the tunneling effect between two different spin compo-

nents. The tunneling currents between spin- $\alpha$ and spin- $\beta(\alpha \neq \beta, \alpha, \beta=-2,-1,0,1,2)$

can be defined by

$$
I_{\alpha \rightarrow \beta}(t)=\frac{d}{d t}\left(P_{\alpha}-P_{\beta}\right)
$$

It is easy to get

$$
\begin{aligned}
& I_{0 \rightarrow \pm 1}(t)=\left(3-\frac{15}{2} \cos ^{2} \lambda\right) \sin (2 \lambda) \frac{d \lambda}{d t}, \\
& I_{0 \rightarrow \pm 2}(t)=\left(\frac{3}{4}-\frac{15}{4} \cos ^{2} \lambda\right) \sin (2 \lambda) \frac{d \lambda}{d t},
\end{aligned}
$$




$$
I_{ \pm 1 \rightarrow \pm 2}(t)=\frac{3}{8}\left(10 \cos ^{2} \lambda-6\right) \sin (2 \lambda) \frac{d \lambda}{d t}
$$

where $\lambda$ is determined by Eq. (7).

Figures 4-6 give the evolutions of the tunneling current with the external magnetic field $b_{l}(t)=b \cos (\omega t)$. The tunneling current among spin- \pm 1 and spin- \pm 2 may exhibit periodically oscillation behavior, but among spin-0 and spin- \pm 1 , spin- 0 and spin- \pm 2 , the tunneling currents exhibit irregular oscillation behavior.

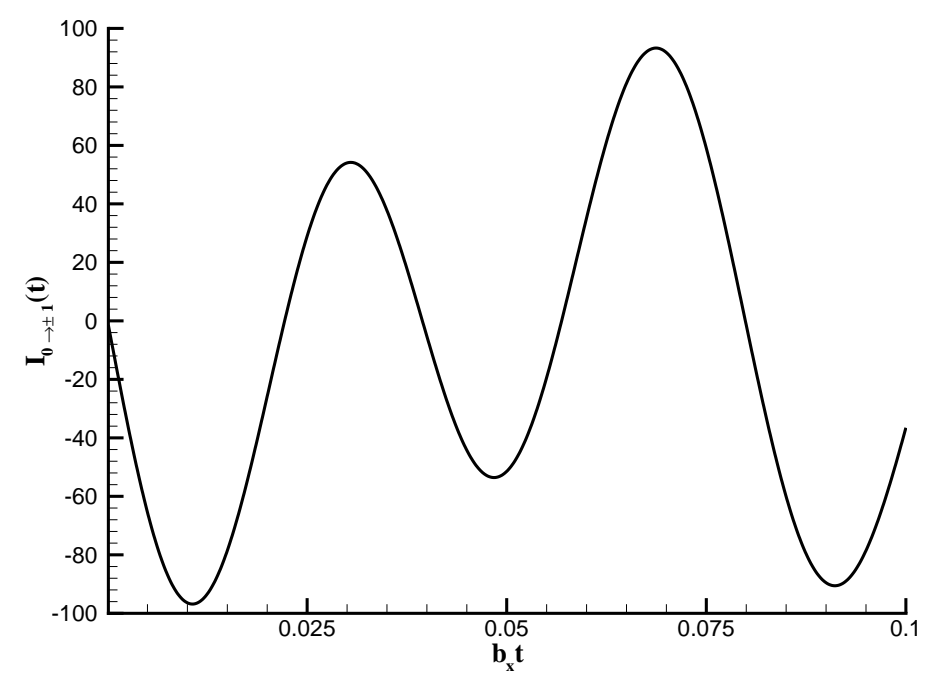

Figure 4: Time evolution of tunneling current $I_{0 \rightarrow \pm 1}(t)$ with an external cosinusoidal magnetic field, $b_{x} / \omega=40, b / \omega=8$. 


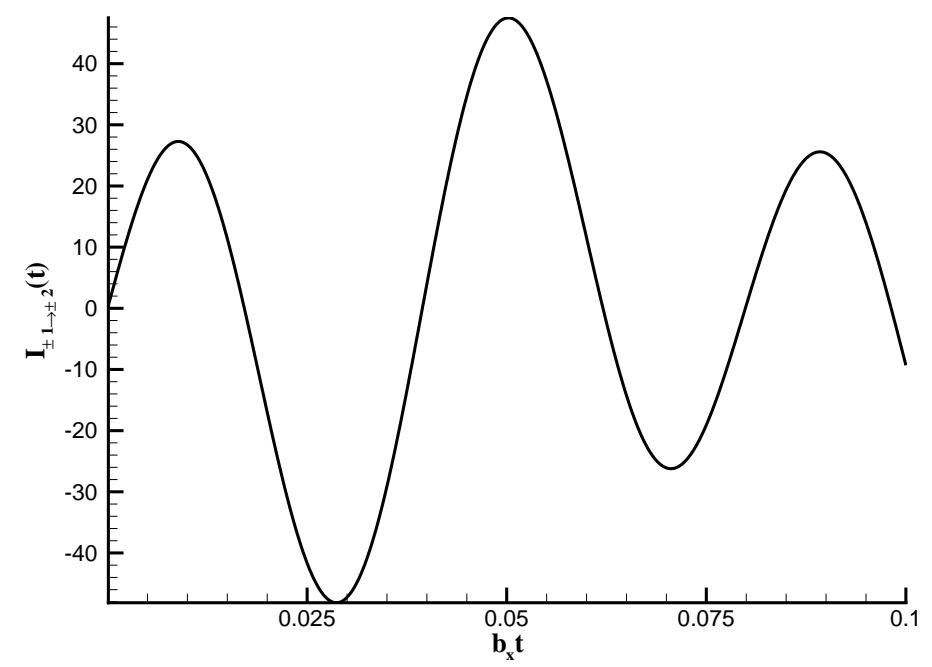

Figure 5: Time evolution of tunneling current $I_{ \pm 1 \rightarrow \pm 2}(t)$ with an external cosinusoidal magnetic field, $b_{x} / \omega=40, b / \omega=8$.

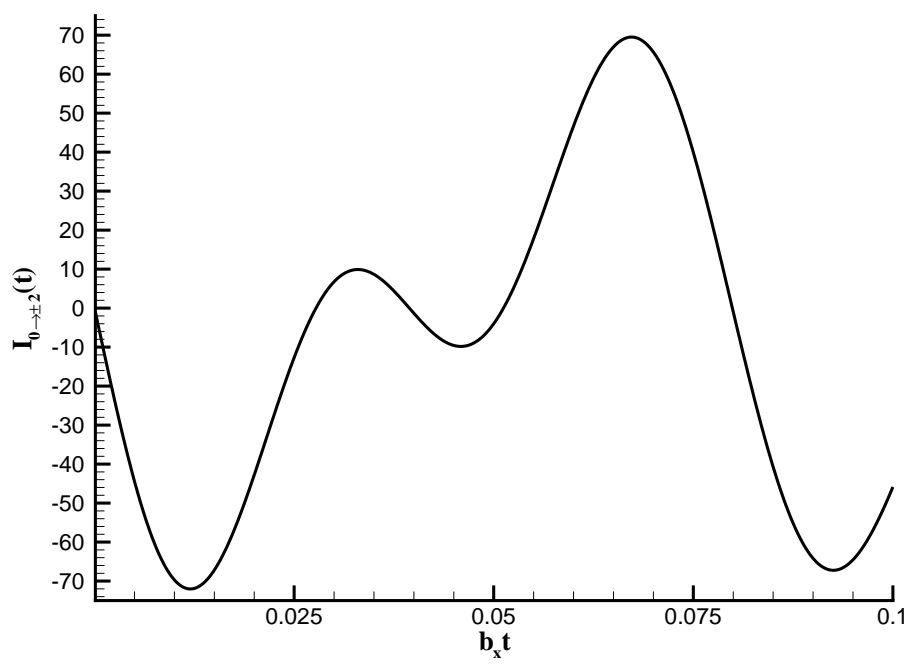

Figure 6: Time evolution of tunneling current $I_{0 \rightarrow \pm 2}(t)$ with an external cosinusoidal magnetic field, $b_{x} / \omega=40, b / \omega=8$. 


\section{Conclusions}

In this paper, we have studied tunneling effect of the spin-2 Bose condensate driven

by external magnetic field $b_{l}(t)=b \cos (\omega t)$. We find that the population transfers among spin- 0 and spin- \pm 1 , spin- 0 and spin- \pm 2 exhibit the step structure under the external cosinusoidal magnetic field respectively, but there do not exist step structure among spin- \pm 1 and spin- \pm 2 . The tunneling current among spin- \pm 1 and spin- \pm 2 may exhibit periodically oscillation behavior, but among spin-0 and spin- \pm 1 , spin-0 and spin- \pm 2 , the tunneling currents exhibit irregular oscillation behavior.

\section{Acknowledgments}

This work was supported by the NSF of Shandong Province.

\section{References}

[1] M. Barrett et al., Phys. Rev. Lett. 87(2001)010404.

[2] N. N. Klausen et al.,Phys.Rev. A64(2001)053602.

[3] J. Stenger et al., Nature 369(1998)345.

[4] T. Ohmi and K.Machida, J.Phys. soc. Jpn. 67(1998)1822. 
[5] T.-L. Ho, Phys. Rev. Lett. 81(1998)742.

[6] C. K. Law, H. Pu, and N. P. Bigelow, Phys. Rev. Lett. 81(1998)5257.

[7] C. V. Ciobanu, S.-K. Yip, and T.-L. Ho, Phys. Rev. A 61(2000)033607.

[8] M. Koashi, and M. Ueda, Phys. Rev. Lett. 84(2000)1066.

[9] P. Zhang, A. Z. Zhang, S. Q. Duan, X. G. Zhao., Phys. Rev. A66(2002)043606. 